\title{
Quantum Darwinism in Quantum Brownian Motion
}

\author{
Robin Blume-Kohout ${ }^{1,2}$ and Wojciech H. Zurek ${ }^{1}$ \\ ${ }^{1}$ Theoretical Division, LANL, Los Alamos, New Mexico 87545, USA \\ ${ }^{2} I Q I$, Caltech, Pasadena, California 91125, USA; \\ Perimeter Institute, Waterloo, Ontario N2L2Y5, Canada
}

(Received 15 May 2007; revised manuscript received 28 March 2008; published 11 December 2008)

\begin{abstract}
Quantum Darwinism - the redundant encoding of information about a decohering system in its environment-was proposed to reconcile the quantum nature of our Universe with apparent classicality. We report the first study of the dynamics of quantum Darwinism in a realistic model of decoherence, quantum Brownian motion. Prepared in a highly squeezed state-a macroscopic superposition-the system leaves records whose redundancy increases rapidly with initial delocalization. Redundancy appears rapidly (on the decoherence time scale) and persists for a long time.
\end{abstract}

PACS numbers: 03.65.Ta, 03.65.Yz, 03.67.Mn, 03.67.Pp

Quantum mechanics is famously difficult to reconcile with everyday classical experience [1-3]. Whereas classical systems have objective properties, quantum systems are disturbed by measurements, so independent observers typically invalidate each others' results. Quantum Darwinism $[2,4-6]$ is a mechanism by which objectivity can emerge within quantum mechanics. It is based on the realization that observers learn about a system $\mathcal{S}$ indirectly, by measuring a fraction of its environment $\mathcal{E}$, so an observable is accessible only when it is redundantly recorded in $\mathcal{E}$. Such indirect observation does not disturb the system, nor invalidate past measurements.

Previous work explored the kinematics of quantum Darwinism-the correlations between $\mathcal{S}$ and its $\mathcal{E}$ that are (a) possible, and (b) typical. We now know the following: (i) that a system can be redundantly correlated with its environment, but only classically (through a single observable) [6]; (ii) that most states of $\mathcal{S E}$ are not correlated this way [5]; and (iii) that simple models of decoherence can produce redundancy [5]. An obvious question remains, "Do realistic models lead to quantum Darwinism?"

We report observation of quantum Darwinism in quantum Brownian motion (QBM), a widely used model of decoherence that couples an oscillator $\mathcal{S}$ to an oscillator bath [7-9]. Prepared in a macroscopic superposition, the system $\mathcal{S}$ rapidly develops massively redundant correlation with the bath (a signature of quantum Darwinism), an effect robust to variation in model parameters. This indicates that objectivity is compatible with quantum theory, and a natural by-product of decoherence. We examine how redundant correlations emerge: how rapidly do they appear, and how long do they persist?

QBM is far richer than simple C-NOT models considered before [5,6], demonstrating dissipation and an unsharp pointer observable (approximate phase space location [9]). Nonetheless, we obtain two surprisingly simple results: a scale-free expression [Eq. (13)] for the mutual information between $\mathcal{S}$ and a fraction of $\mathcal{E}$; and an even simpler formula for redundancy [Eq. (14)] as a function of the system's initial delocalization (e.g., initial squeezing $s$ ) and the information deficit $\delta$ quantifying the observer's residual uncertainty.

Background-When a system decoheres, information is recorded in the environment $\mathcal{E}[2,10]$. Proliferation of this information-repeated copying in subsystems of $\mathcal{E}$-is the essence of quantum Darwinism. This does not contradict the no-cloning theorem [11]; at most one observable can be redundantly recorded in $\mathcal{E}$ [6]. Simultaneous recording of two noncommuting observables, in different places, would violate Heisenberg's uncertainty principle. Thus, the dynamics of the $\mathcal{S}-\mathcal{E}$ interaction that generates records (even imperfect ones) in the environment must break unitary invariance and single out a preferred basis. The dynamics select one observable (at most) to be redundantly recorded. Complementary observables get recorded in entangled modes of $\mathcal{E}$, inaccessible to any local observer. This propagation of information about the fittest observable throughout the environment, at the expense of complementary observables, is quantum Darwinism.

We look for it in quantum Brownian motion (QBM). A common model for a system coupled weakly to a large bath, QBM describes an oscillator $\left(\mathbf{H}_{\text {sys }}=\frac{p_{\mathcal{S}}^{2}}{2 m_{\mathcal{S}}}+\frac{m_{\mathcal{S}} \Omega_{0}^{2} x_{\mathcal{S}}^{2}}{2}\right)$, its position $x_{\mathcal{S}}$ linearly coupled to a bath of oscillators $\mathcal{E}_{\omega}$, each with frequency $\omega$ and coordinates $y_{\omega}$ and $q_{\omega}$. The Hamiltonian is

$$
\mathbf{H}=\mathbf{H}_{\mathrm{sys}}+\frac{1}{2} \sum_{\omega}\left(\frac{q_{\omega}^{2}}{m_{\omega}}+m_{\omega} \omega^{2} y_{\omega}^{2}\right)+x_{\mathcal{S}} \sum_{\omega} C_{\omega} y_{\omega} .
$$

The coupling strengths $C_{\omega}$ are encapsulated in a spectral density, $I(\omega)=\sum_{n} \delta\left(\omega-\omega_{n}\right) \frac{C_{n}^{2}}{2 m_{n} \omega_{n}}$. We consider an Ohmic bath, at zero temperature, with a cutoff $\Lambda$, so $I(\omega)=\frac{2 m_{\mathcal{S}} \gamma_{0}}{\pi} \omega$ for $\omega \in[0 \ldots \Lambda]$. For our continuum bath, the coupling to $\mathcal{E}_{\omega}$ is a differential element $d C_{\omega}^{2}=$ $\frac{4}{\pi} m_{\mathcal{S}} m_{\omega} \gamma_{0} \omega^{2} d \omega$, but for numerics, we divide $\mathcal{E}$ into 1024 discrete bands of width $\Delta \omega\left(\right.$ a good model until $\left.t \sim \frac{2 \pi}{\Delta \omega}\right)$.

The system $\mathcal{S}$ is a massive (macroscopic) underdamped harmonic oscillator. We choose units where $\hbar=1$, the bath 
masses are $m_{\omega}=1$, and the system's renormalized frequency is $\Omega=4$. In these units, bath frequencies lie in $[0 \ldots \Lambda=16]$, and we vary the coupling $\gamma_{0}$ with $m_{\mathcal{S}}$ so that $m_{\mathcal{S}} \gamma_{0}=25$. Generally, $m_{\mathcal{S}}=10^{3}$ and $\gamma_{0}=\frac{1}{40}$. $\mathcal{S}$ is prepared in a squeezed Gaussian state (parametrized by $s_{x}=s_{p}^{-1} \equiv \frac{\Delta x}{\Delta p}$, where $s_{x}=s_{p}=1$ for the ground state). This is a superposition of macroscopically separated positions (for $s_{x} \gg 1$ ) or momenta (for $s_{p} \gg 1$ ), and demonstrates nonlocal coherence like a "Schrödinger's cat" state.

We evolve the joint state of $\mathcal{S}$ and $\mathcal{E}$ in time, then analyze it for redundant correlations. Interaction produces entanglement between $\mathcal{S}$ and $\mathcal{E}$, decohering $\mathcal{S}$ itself. The system's entropy rises (as superposition is converted into mixture), as does its correlation with $\mathcal{E}$. Thus, $\mathcal{E}$ is not merely a sink for lost information, but a witness from which information about $\mathcal{S}$ can be obtained. Only when $\mathcal{S}$ is strongly correlated with many disjoint fragments $\mathcal{F}$ of $\mathcal{E}$ (Fig. 1) can many independent observers learn about $\mathcal{S}$ without disturbing it (objectivity). We measure correlation of $\mathcal{S}$ and $\mathcal{F}$ with quantum mutual information, an upper bound for the decrease in $H_{\mathcal{S}}$ due to a measurement of $\mathcal{F}$,

$$
I_{\mathcal{S}: \mathcal{F}}=H_{\mathcal{S}}+H_{\mathcal{F}}-H_{\mathcal{S F}},
$$

where $H_{i}$ is the von Neumann entropy of $\rho_{i}$.

To identify redundant correlations, we examine the partial information provided by a random fragment that contains a fraction $f$ of $\mathcal{E}$ 's bandwidth,

$$
\bar{I}(f)=\operatorname{avg}_{\text {all }} \mathcal{F} \text { of size } f\left(I_{\mathcal{S}: \mathcal{F}}\right) .
$$

Partial information plots (PIPs) for joint pure states of $\mathcal{S}$ and $\mathcal{E}$ are always reflection symmetric (see [5], and Fig. 2). They assume a characteristic shape in the presence of redundancy: $\bar{I}(f)$ increases sharply at $f=0$ and $f=1$, with a wide, flat "classical plateau" in between [5]. This shows that a small fraction $f_{\delta}$ of $\mathcal{E}$ provides all but $\delta$ of the classical information, while complementary information is

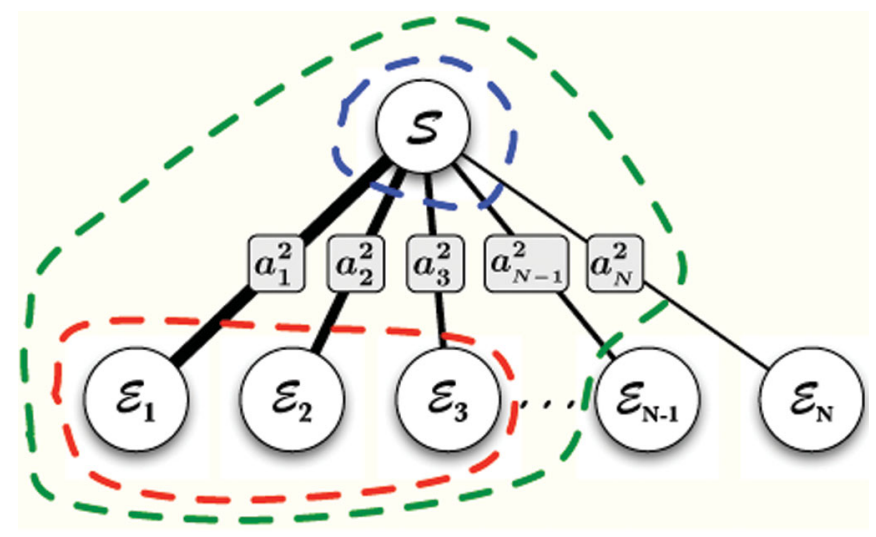

FIG. 1 (color). Information about the system $(\mathcal{S})$ is available from fragments of the bath $(\mathcal{E})$. The QBM $\mathcal{E}$ is composed of many bands $\left(\mathcal{E}_{\omega}\right)$ each represented by an oscillator with frequency $\omega$. Interaction produces correlated joint states of $\mathcal{S E}: \mathcal{S}$ develops independent correlations with each band (black lines), increasing by $a_{k}^{2}$ the phase space volume of $\mathcal{S}$ and $\mathcal{E}_{\omega}$. A fragment $\mathcal{F}$ (red) comprises several noncontiguous bands. inaccessible except by capturing nearly all of $\mathcal{E}$. The plateau's slope quantifies the nonredundant information, determining $\delta$.

To quantify redundancy, we define "information about $\mathcal{S}$ is $R$-fold redundant" to mean that $R$ disjoint fragments of $\mathcal{E}$ are each nearly as strongly correlated (to within $\delta$; see below) with $\mathcal{S}$ as is the entire bath. The bath must factor into $\mathcal{E}=\mathcal{F}_{1} \otimes \mathcal{F}_{2} \otimes \ldots \mathcal{F}_{R}$ such that, for each fragment $\mathcal{F}_{i}, I_{\mathcal{S}: \mathcal{F}_{i}} \approx H_{\mathcal{S}}$. We allow a residual uncertainty of $\delta$, so $I_{\mathcal{S}: \mathcal{F}} \geq(1-\delta) H_{\mathcal{S}}$, and report $R_{\delta}$,

$$
R_{\delta}=1 / f_{\delta}
$$

where $f_{\delta}$ (the fraction of $\mathcal{E}$ 's bandwidth that $\mathcal{F}$ must contain to ensure $\left.I_{\mathcal{S}: \mathcal{F}} \geq(1-\delta) H_{\mathcal{S}}\right)$ is determined via Monte Carlo calculations.

Exact solutions for QBM [8] provide only the reduced state of $\mathcal{S}$. We use numerics to obtain exact solutions for a 1024-oscillator $\mathcal{E}$, and compare them with a simple theory. The combined system begins in a Gaussian state $(\mathcal{S}$ in a squeezed state, $\mathcal{E}$ in its ground state), described by its mean and variance,

$$
\vec{z}=\left(\begin{array}{c}
\langle x\rangle \\
\langle p\rangle
\end{array}\right) ; \quad \Delta=\left(\begin{array}{cc}
\Delta x^{2} & \Delta x p \\
\Delta x p & \Delta p^{2}
\end{array}\right),
$$
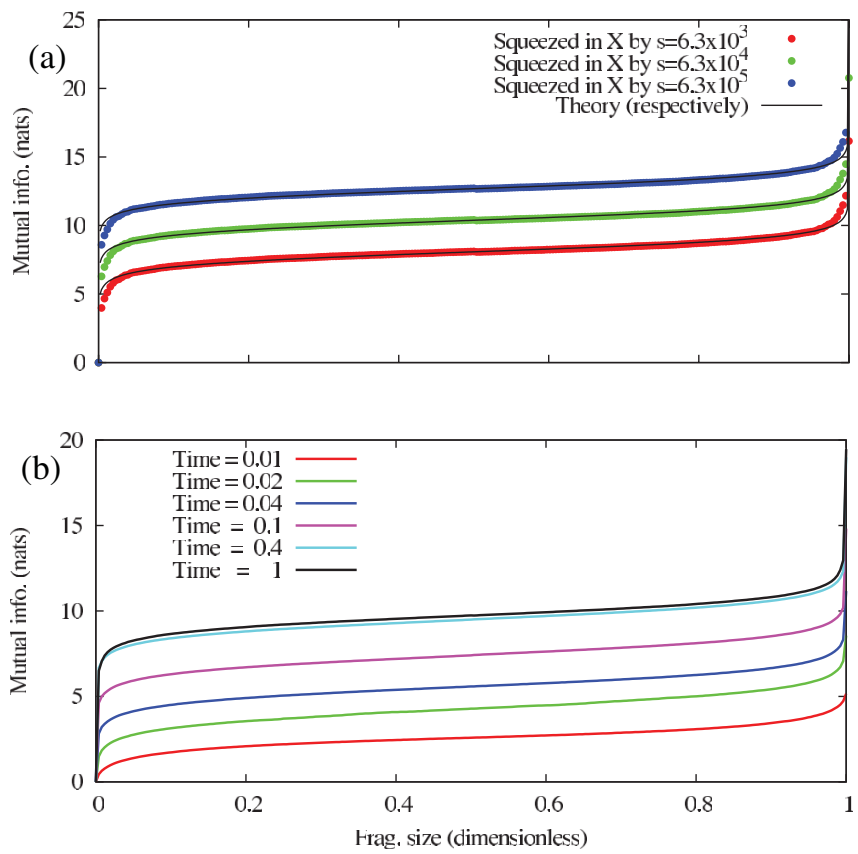

FIG. 2 (color). Partial information plots (PIPs) show how information about $\mathcal{S}$ is stored in $\mathcal{E}$, plotting $I_{\mathcal{S}: \mathcal{F}}$ (information in a random fragment $\mathcal{F} \subset \mathcal{E}$ ) against $\mathcal{F}$ 's size $(f)$. PIPs are symmetric when the joint state is pure [5]. We initialized $\mathcal{S}$ in an $x$-squeezed state, which evolves into a superposition of $|x\rangle$ states that decohere. Plot (a) shows PIPs at $t=4\left(\tau_{\text {decoherence }} \approx 0.1\right)$, for three different values of squeezing. Small fragments provide most of the available information about $\mathcal{S}$; squeezing changes the amount of redundant information, not the overall shape of the PIP. Numerics (dots) agree with a simple theory (lines). Plot (b) tracks one state during decoherence $(t=0.01, \ldots, 4)$, and confirms the PIPs' invariant shape. 
where $\vec{z}$ is irrelevant to correlations, and the obvious generalization for $N$-mode states (to a $2 N$-dimensional phase space) holds. QBM's linear dynamics preserve Gaussian form, so we can evolve $\Delta(t)$ using canonical methods. Mutual information between subsystems (e.g., $\mathcal{S}$, a band $\mathcal{E}_{\omega}$, or a fragment $\mathcal{F}$ ) is calculated from the reduced states' entropy. For Gaussian states, $H(\rho)$ is a function of squared symplectic area,

$$
\begin{gathered}
a^{2}=\left(\frac{\hbar}{2}\right)^{-2} \operatorname{det}(\Delta), \\
H(a)=\frac{1}{2}\left(\begin{array}{c}
(a+1) \ln (a+1) \\
-(a-1) \ln (a-1)
\end{array}\right)-\ln 2 \approx \ln \left(\frac{e}{2} a\right),
\end{gathered}
$$

where the approximation is excellent for $a>2$. For multiple-mode fragments, $H(\rho)$ is a sum over $\Delta$ 's symplectic eigenvalues $a_{i}^{2}$ [12]. We do this exactly in numerics, but in theory calculations we apply the single-mode formula to $a^{2}=\prod_{i} a_{i}^{2}$.

Partial information plots at several times (Fig. 2) show that small fragments provide almost as much information as large ones; all but $\sim \ln 2$ bits of information about $\mathcal{S}$ is redundant. When $\mathcal{S}$ is macroscopic, this nonredundant information is dwarfed by the total information. Very large fragments provide some unique information $[\bar{I}(f)$ rises sharply at $f=1]$ —an observer who captures every subsystem of $\mathcal{E}$ can measure nonpointer observables. Avoiding classicality is absurdly difficult.

Just how redundant is the information about $\mathcal{S}$ ? Figure 3 shows how redundancy evolves over time, in several scenarios. Our main results are the following: (i) substantial redundancy appears; (ii) it appears rapidly and persists thereafter; and (iii) redundancy grows rapidly with the spatial extent of the system's initial state. Many fragments of $\mathcal{E}$ know the location of $\mathcal{S}$ to within microscopic error, making this information effectively objective. Redundancy appears on the decoherence time scale, not the (much longer) dissipation time scale. When $\left|\psi_{0}\right\rangle$ is extended in $\hat{x}$ [Fig. 3(b)], decoherence and redundancy appear very quickly $\left(\tau \sim \Lambda^{-1}\right)$. If $\left|\psi_{0}\right\rangle$ is extended in $\hat{p}$ [Fig. 3(a)], then the system's oscillation prepares a superposition of $|x\rangle$ states adiabatically (the physically relevant scenario), and $R_{\delta}$ rises over a quarter period $\left(\tau \sim \Omega^{-1}\right)$. Dissipation, surprisingly, appears to increase redundancy [see Fig. 3(d), and the concluding paragraph].

Theory.-A simple theory based on the BornOppenheimer approximation predicts most of these results. When $\mathcal{S}$ is very massive $\left(m_{\mathcal{S}} \rightarrow \infty\right.$, implying $\left.\gamma_{0} \ll 1\right)$, the backreaction of $\mathcal{E}_{\omega}$ on $\mathcal{S}$ is very small, and $\mathcal{S}$-mediated interactions between bath modes are negligible. $\mathcal{S}$ exerts a sinusoidal driving force on the bath oscillators, and develops independent correlations with each bath mode $\mathcal{E}_{\omega}$, quantified by its squared symplectic area $\left(a^{2} \propto \Delta y^{2} \Delta q^{2}\right)$. We begin by ignoring $\mathbf{H}_{\text {sys }}$ entirely (as $m_{\mathcal{S}} \rightarrow \infty$, it nearly commutes with the interaction) to get the correct form, then restore $\mathbf{H}_{\text {sys }}$ and resolve the equation of motion.

Neglecting $\mathbf{H}_{\mathrm{sys}}$ yields $\mathbf{H}=\sum_{\omega} \mathbf{H}_{\omega}+C_{\omega}\left(x_{\mathcal{S}} \otimes y_{\omega}\right)$. Each $\mathcal{E}_{\omega}$ feels a conditional Hamiltonian $\mathbf{H}_{\omega}\left(x_{\mathcal{S}}\right)$, and
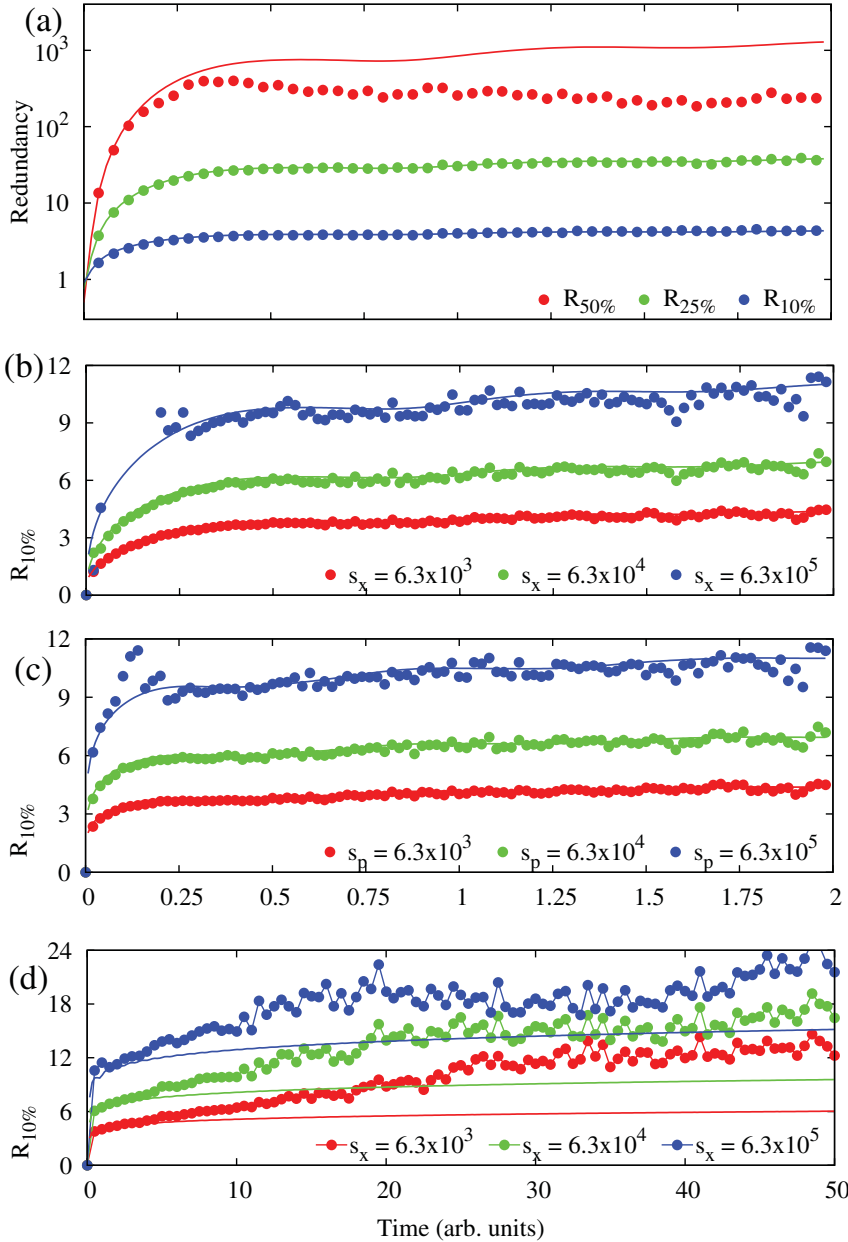

FIG. 3 (color). Delocalized states of a decohering oscillator $(\mathcal{S})$ are redundantly recorded by the environment $(\mathcal{E})$. In plot (a), $\mathcal{S}$ is initially squeezed in $x$ by $s_{x}=6.3 \times 10^{3}$, and we see that redundancy $\left[R_{\delta}(t)\right]$ increases exponentially with the information deficit $\delta$ (as $\left.R_{\delta} \approx s^{2 \delta}\right)$. $R_{10 \%} \sim 10$ may seem modest, but $\delta=$ $10 \%$ implies resolving $x$ to within $\sim 3$ ground-state widths, comparable to experiments in nanomechanical resonators [13]. At $\delta \sim 0.5, \mathcal{E}$ resolves $\sim \sqrt{s}$ different locations, and $R_{50 \%} \gtrsim 10^{3}$ saturates our numerical resolution. Plots (b)-(d) show that $R_{10 \%}$-redundancy of $90 \%$ of the available information-grows with the initial squeezing $\left(s_{x}\right.$ or $\left.s_{p}\right)$. Dots denote numerics; lines-our theory. Redundancy develops with decoherence: $p$-squeezed states (c) decohere almost instantly, while $x$-squeezed states (a),(b) decohere only as they evolve into $p$-squeezed states. Redundancy persists thereafter (d), and by $t \sim O\left(\gamma^{-1}\right)$ dissipation boosts $R_{10 \%}$ above our simple theory.

evolves as $\left|\psi_{\omega}(0)\right\rangle \rightarrow\left|\psi_{\omega}\left(t ; x_{\mathcal{S}}\right)\right\rangle$, conditional upon $x_{\mathcal{S}}$. If $\left|\psi_{\mathcal{S}}(0)\right\rangle$ is a superposition of $|x\rangle$ states, this yields a Gaussian singly branching state (see Ref. [5]):

$$
\left(\int \psi_{\mathcal{S}}(x)|x\rangle d x\right) \otimes\left|\psi_{1}(0)\right\rangle\left|\psi_{2}(0)\right\rangle \ldots\left|\psi_{N_{\mathrm{env}}}(0)\right\rangle
$$

$\int \psi_{\mathcal{S}}(x)|x\rangle \otimes\left|\psi_{1}(t ; x)\right\rangle\left|\psi_{2}(t ; x)\right\rangle \ldots\left|\psi_{N_{\mathrm{env}}}(t ; x)\right\rangle d x$. 
The reduced state $\rho_{\mathcal{A}}$ for a subsystem $\mathcal{A}$ is spectrally equivalent to a partially decohered state for $\mathcal{S}: \rho_{\mathcal{A}}\left(x, x^{\prime}\right)=$ $\rho_{\mathcal{S}}\left(x, x^{\prime}, t=0\right) \Gamma_{\mathcal{A}}\left(x, x^{\prime}\right)$, where $\Gamma_{\mathcal{A}}\left(x, x^{\prime}\right)$ is a product (over all $\mathcal{E}_{\omega} \notin \mathcal{A}$ if $\mathcal{A}$ contains $\mathcal{S}$, or all $\mathcal{E}_{\omega} \in \mathcal{A}$ otherwise) of $\Gamma_{\omega}\left(x, x^{\prime}\right) \equiv\left\langle\psi_{\omega}(t ; x) \mid \psi_{\omega}\left(t ; x^{\prime}\right)\right\rangle$.

When $\mathcal{S}$ is in state $|x\rangle, \mathcal{E}_{\omega}$ feels a displaced oscillator Hamiltonian

$$
\mathbf{H}_{\omega}(x)=\frac{q_{\omega}^{2}}{2 m_{\omega}}+\frac{m_{\omega} \omega^{2}}{2}\left(y_{\omega}-\delta y_{\omega}\right)^{2}-\frac{m_{\omega}^{2} \omega^{2}}{2} \delta y_{\omega}^{2}
$$

centered at $\delta y_{\omega}=C_{\omega} x_{\mathcal{S}} /\left(m_{\omega} \omega^{2}\right)$. Solving the equation of motion and inserting $\Delta y_{0}^{2}=\frac{\hbar}{2 m_{\omega}}, \Delta q_{0}^{2}=\frac{\hbar m_{\omega}}{2}$ yields

$$
\left|\Gamma_{x, x^{\prime}}^{(\omega)}\right|=\exp \left[-\frac{C_{\omega}^{2}}{2 m_{\omega} \hbar \omega^{3}}\left(x-x^{\prime}\right)^{2}(1-\cos \omega t)\right] .
$$

The exponent being proportional to $\left(x-x^{\prime}\right)^{2}$, we define a decoherence factor, $D_{\omega}(t) \equiv-\log \left(\Gamma_{\omega}\left(x, x^{\prime}\right)\right) /\left(x-x^{\prime}\right)^{2}$. Because $\mathcal{E}$ has continuous spectral density, $D_{\omega}$ is a differential $d D_{\omega}=\frac{2 m_{\mathcal{S}} \gamma_{0}}{\pi \hbar \omega}(1-\cos \omega t) d \omega$, and $D_{\mathcal{A}}$ for subsystem $\mathcal{A}$ is an integral.

We now reinsert $\mathbf{H}_{\mathcal{S}}$ and resolve Eq. (9) to get

$$
\frac{d D}{d \omega}=\frac{m_{\mathcal{S}} \gamma_{0}}{\pi \hbar} \frac{\omega^{3} d \omega}{\left(\Omega^{2}-\omega^{2}\right)^{2}}\left[\begin{array}{c}
\left(\sin \omega t-\frac{\Omega}{\omega} \sin \Omega t\right)^{2} \\
+(\cos \omega t-\cos \Omega t)^{2}
\end{array}\right] .
$$

This quantifies the decohering power of band $\mathcal{E}_{\omega}$. Contributions from high-frequency bands are suppressed by $\omega^{-1}$, while resonant bands near $\omega \sim \Omega$ dominate. Results are thus largely independent of the cutoff.

Decoherence suppresses the off-diagonals of $\rho\left(x, x^{\prime}\right)$, leaving $\Delta x^{2}$ unchanged but increasing $\Delta p^{2}$ by $\delta p_{\mathcal{A}}^{2}=$ $2 \hbar D_{\mathcal{A}}$. A subsystem's squared symplectic area increases by

$$
\begin{aligned}
a_{\mathcal{A}}^{2} & \rightarrow 1+\delta a_{\mathcal{A}}^{2}=1+\left(\frac{\hbar}{2}\right)^{-2} \Delta x^{2} \delta p_{\mathcal{A}}^{2} \\
& =1+\frac{8 \Delta x^{2}}{\hbar} D_{\mathcal{A}} .
\end{aligned}
$$

For multimode subsystems, $\delta a^{2}$ is the integral $\delta a_{\mathcal{S}}^{2}=$ $\frac{8 \Delta x^{2}}{\hbar} \int_{0}^{\Lambda} \frac{d D}{d \omega} d \omega$. The integral for $\delta a_{\mathcal{F}}^{2}$ is over all $\mathcal{E}_{\omega} \in \mathcal{F}$, for $\delta a_{\mathcal{S} \mathcal{F}}^{2}$ over all $\mathcal{E}_{\omega} \notin \mathcal{F}$, and for $\delta a_{\mathcal{S}}^{2}$ over all of $\mathcal{E}$ (see Fig. 1).

Discussion.-Each bath mode has [nearly] independent correlations with $\mathcal{S}$. Thus, when $\mathcal{F}$ contains a fraction $f$ of $\mathcal{E}$ 's bandwidth, $a_{\mathcal{F}}^{2}=1+f \delta a_{\mathcal{S}}^{2}$ and $a_{\mathcal{S F}}^{2}=1+(1-$ f) $\delta a_{\mathcal{S}}^{2}$. Mutual information follows from Eq. (7),

$$
I_{\mathcal{S}: \mathcal{F}} \approx H_{\mathcal{S}}+\frac{1}{2} \ln \left(\frac{f}{1-f}\right)
$$

This simple result fits numerics well (see Fig. 2) and predicts the invariant shape of partial information plots.

To compute redundancy, we count disjoint fragments with $I_{\mathcal{S}: \mathcal{F}} \geq(1-\delta) H_{\mathcal{S}}$. As $I_{\mathcal{S}: \mathcal{F}}$ depends only on the size $f, I_{\mathcal{S}: \mathcal{F}} \geq(1-\delta) H_{\mathcal{S}}$ iff $f \geq f_{\delta}=\frac{e^{-2 \delta H_{\mathcal{S}}}}{1+e^{-2 \delta H_{\mathcal{S}}}} . \mathcal{E}$ contains $1 / f_{\delta}$ such fragments, and an $s$-squeezed state deco- heres to a mixed state with $H_{\mathcal{S}} \approx \ln s[2,8]$, so

$$
R_{\delta} \approx e^{2 \delta H_{\mathcal{S}}} \approx s^{2 \delta} .
$$

This expression is a succinct summary of our results-and fits the data remarkably well (Fig. 3). For instance, setting $\delta=0.5$ means localizing $\mathcal{S}$ to within $\sim \sqrt{s}$, with redundancy $R_{0.5} \propto s$ [Fig. 3(a)].

To generalize beyond squeezed states, note that $R_{\delta}$ increases rapidly with the spatial extent $\Delta x_{\mathcal{S}}$ of $\mathcal{S}$ 's wave function. A fragment of $\mathcal{E}$ provides a fuzzy record of $\mathcal{S}$ 's position whose resolution increases with $\mathcal{F}$ 's size. A true Schrödinger's cat state will yield high redundancy (but only $\sim 1$ bit of entropy), as small fragments can resolve the two branches. As $\mathcal{S}$ oscillates, $\mathcal{E}$ records both $\hat{x}$ and $\hat{p}$. Thus, QBM (unlike the model of Ref. [5]) inscribes an unsharp observable - approximate phase space location [9] - throughout $\mathcal{E}$. Complementary information-relative phase between branches of a Schrödinger's cat state-is hidden from realistic observers.

This is quantum Darwinism, but with some novel features. The system's constant oscillation forces $\mathcal{E}$ to record an unsharp observable. This information becomes rapidly redundant, although 1 bit (the slightly higher resolution provided by larger fragments) remains nonredundant. Backreaction of $\mathcal{E}$ on $\mathcal{S}$ causes dissipation, reducing correlation by $t \sim \gamma_{0}^{-1}$. Simulations (confirmed by more detailed calculations) show that "nonredundant" correlations disappear first, enhancing redundancy [see Fig. 3(d)]. Thus, proliferation of information about one observable is followed by rapid disappearance of all other information.

We thank D. Poulin for discussions, and DOE for support via LDRD at Los Alamos.

[1] J.A. Wheeler and W.H. Zurek, Quantum Theory and Measurement (Princeton University, Princeton, NJ, 1983).

[2] W. H. Zurek, Rev. Mod. Phys. 75, 715 (2003).

[3] M. Schlosshauer, Rev. Mod. Phys. 76, 1267 (2005); M. Schlosshauer, Decoherence and the Quantum-to-Classical Transition (Springer, New York, 2007).

[4] W. H. Zurek, arxiv:0707.2832.

[5] R. Blume-Kohout and W.H. Zurek, Phys. Rev. A 73, 062310 (2006); Found. Phys. 35, 1857 (2005).

[6] H. Ollivier et al., Phys. Rev. Lett. 93, 220401 (2004); H. Ollivier et al., Phys. Rev. A 72, 042113 (2005).

[7] A. Caldeira and A. Leggett, Physica (Amsterdam) 121A, 587 (1983).

[8] B. L. Hu et al., Phys. Rev. D 45, 2843 (1992).

[9] W. H. Zurek et al., Phys. Rev. Lett. 70, 1187 (1993).

[10] J. J. Halliwell, Phys. Rev. D 60, 105031 (1999); M. GellMann and J.B. Hartle, in Quantum Classical Correspondence, edited by D.-H. Feng and B.-L. Hu (International Press, Boston, 1997).

[11] W. K. Wootters and W. H. Zurek, Nature (London) 299, 802 (1982); D. Dieks, Phys. Lett. A 92, 271 (1982).

[12] A. Serafini et al., J. Phys. B 37, L21 (2004).

[13] M. D. LaHaye et al., Science 304, 74 (2004). 